\title{
NORMAL FAMILIES OF MEROMORPHIC FUNCTIONS WITH MULTIPLE POLES
}

\author{
YUNTONG LI
}

\begin{abstract}
Let $\mathscr{F}$ be a family of meromorphic functions defined in a domain $\mathscr{D}$, and $a, b$ be two constants such that $a \neq 0, \infty$ and $b \neq \infty$. If for each $f \in \mathscr{F}$, all poles of $f(z)$ are of multiplicity at least 3 in $\mathscr{D}$, and $f^{\prime}(z)+a f^{2}(z)-b$ has at most 1 zero in $\mathscr{D}$, ignoring multiplicity, then $\mathscr{F}$ is normal in $\mathscr{D}$.
\end{abstract}

\section{Introduction and main results}

Let $\mathscr{D}$ be a domain in $\mathbb{C}$, and $\mathscr{F}$ be a family of meromorphic functions defined in the domain $\mathscr{D}$. $\mathscr{F}$ is said to be normal in $\mathscr{D}$, in the sense of Montel, if for every sequence $\left\{f_{n}\right\} \subseteq$ $\mathscr{F}$ contains a subsequence $\left\{f_{n_{j}}\right\}$ such that $f_{n_{j}}$ converges spherically uniformly on compact subsets of $\mathscr{D}$ (See [1, Definition 3.1.1]).

$\mathscr{F}$ is said to be normal at a point $z_{0} \in \mathscr{D}$ if there exists a neighborhood of $z_{0}$ in which $\mathscr{F}$ is normal. It is well known that $\mathscr{F}$ is normal in a domain $\mathscr{D}$ if and only if it is normal at each of its points (see [1, Theorem 3.3.2]).

Let $f(z)$ and $g(z)$ be two meromorphic functions in a domain $\mathscr{D} \subseteq \mathbb{C}$, and let $a$ be a finite complex number. If $f(z)-a$ and $g(z)-a$ assume the same zeros, then we say that $f$ and $g$ share the value $a$ in $\mathscr{D}$ IM (ignoring multiplicity)(see [2, pp.115-116]).

In 1959, W. K. Hayman [3] proved the following well-known result.

Theorem A. Let $f$ be a non-constant meromorphic function in the complex plane $\mathbb{C}, n$ be $a$ positive integer and $a, b$ be two constants such that $n \geqslant 5, a \neq 0, \infty$ and $b \neq \infty$. If $f^{\prime}-a f^{n} \neq b$, then $f$ is a constant.

Corresponding to Theorem A there are the following theorems which confirmed a Hayman's well-known conjecture about normal families in [4, Problem 5.14].

Received March 9, 2013, accepted April 9, 2014.

2010 Mathematics Subject Classification. 30D35, 30D45.

Key words and phrases. Meromorphic functions, normal family, multiplicity. 
Theorem B. Let $\mathscr{F}$ be a family of meromorphic functions in a complex domain $\mathscr{D}, n$ be a positive integer and $a, b$ be two constants such that $n \geqslant 3, a \neq 0, \infty$ and $b \neq \infty$. If $f^{\prime}-a f^{n} \neq b$, then $\mathscr{F}$ is normal in $\mathscr{D}$.

Theorem C. Let $\mathscr{F}$ be a family of holomorphic functions in a complex domain $\mathscr{D}, n$ be a positive integer and $a, b$ be two constants such that $n \geqslant 2, a \neq 0, \infty$ and $b \neq \infty$. If $f^{\prime}-a f^{n} \neq b$, then $\mathscr{F}$ is normal in $\mathscr{D}$.

Theorem B is due to S. Li [5, $n \geqslant 5]$, X. Li [6, $n \geqslant 5]$, J. Langley [7, $n \geqslant 5]$, X. Pang [8, $n=4]$, H. Chen and M. Fang [9, $n=3$ ] and L. Zalcman [10, $n=3$ ] independently. Theorem C is due to D. Drasin [11, $n \geqslant 3]$ and Y. Ye [12, $n=2]$.

Generally speaking, for $n=2$, the result of Theorem B is not valid. For examples we refer the reader to [13]. However, in [13], M. Fang and W. Yuan had

Theorem D. Let $\mathscr{F}$ be a family of meromorphic functions in a complex domain $\mathscr{D}$, and $a \neq 0, \infty$, $b \neq \infty$. If, for every $f \in \mathscr{F}, f^{\prime}-a f^{2} \neq b$ and the poles of $f$ are of multiplicity at least 3 , then $\mathscr{F}$ is normal in $\mathscr{D}$.

It is natural to ask whether the condition in Theorem $\mathrm{D}$ that $f^{\prime}-a f^{2} \neq b$ can be relaxed. In this paper we investigate this problem and prove the following result.

Theorem 1. Let $\mathscr{F}$ be a family of meromorphic functions defined in a domain $\mathscr{D}$, and $a, b$ be two constants such that $a \neq 0, \infty$ and $b \neq \infty$. If for each $f \in \mathscr{F}$, all poles of $f(z)$ are of multiplicity at least 3 in $\mathscr{D}$, and $f^{\prime}(z)+a f^{2}(z)-b$ has at most 1 zero in $\mathscr{D}$, ignoring multiplicity, then $\mathscr{F}$ is normal in $\mathscr{D}$.

By the idea of shared values, Q. Zhang [14, Theorem 2] proved.

Theorem E. Let $\mathscr{F}$ be a family of holomorphic functions defined in a domain $\mathscr{D}$ and $a, b$ be two constants such that $a \neq 0, \infty$ and $b \neq \infty$. If for every pair of functions $f, g \in \mathscr{F}, f^{\prime}+a f^{2}$ and $g^{\prime}+a g^{2}$ share the value $b$ in $\mathscr{D}$, then $\mathscr{F}$ is normal in $\mathscr{D}$.

It is natural to ask whether Theorems D can be improved by the idea of shared values. In this paper, we study the problem and obtain the following theorem.

Theorem 2. Let $\mathscr{F}$ be a family of meromorphic functions defined in a domain $\mathscr{D}$. Let $a \neq 0, b$ be two finite complex number. If for each $f \in \mathscr{F}$, all poles of $f(z)$ are of multiplicity at least 3 in $\mathscr{D}$, and if $f^{\prime}+a f^{2}$ and $g^{\prime}+a g^{2}$ share the value $b$ in $\mathscr{D}$ for every pair of functions $f, g \in \mathscr{F}$, then $\mathscr{F}$ is normal in $\mathscr{D}$.

Example 1. Let $\mathscr{D}=\{z:|z|<1\}$. Let $\mathscr{F}=\left\{f_{m}\right\}$ where $f_{m}:=\frac{1}{m z^{k}}$, then $f_{m}^{\prime}+f^{2}=\frac{1-k m z^{k-1}}{m^{2} z^{2 k}}$, so $f_{m}^{\prime}+f^{2}$ has exactly one zero for $k=2$ and $f_{m}^{\prime}+f^{2}$ has two distinct zeros for $k=3$. However, it is easily obtained that $\mathscr{F}$ is not normal at the point $z=0$. 
This shows that the condition that all poles of $f(z)$ are of multiplicity at least 3 and $f^{\prime}(z)+$ $a f^{2}(z)$ has at most 1 zero in Theorems 1 is sharp.

\section{Some lemmas}

To prove our results, we need some preliminary results.

Lemma 1 ([15], [16] Lemma 1 (Zalcman's Lemma)). Let $\mathscr{F}$ be a family of functions meromorphic on a domain $\mathscr{D}$, all of whose poles have multiplicity at least $j$; Then if $\mathscr{F}$ is not normal at $z_{0} \in \mathscr{D}$, there exist, for each $j<\alpha<1$,

(a) points $z_{n}, z_{n} \rightarrow z_{0}$;

(b) functions $f_{n} \in \mathscr{F}$; and

(c) positive numbers $\rho_{n} \rightarrow 0^{+}$

such that $\rho_{n}^{\alpha} f_{n}\left(z_{n}+\rho_{n} \xi\right)=g_{n}(\xi) \rightarrow g(\xi)$ locally uniformly with respect to the spherical metric, where $g(\xi)$ is a nonconstant meromorphic function on $\mathbb{C}$. Moreover, the order of $g(\xi)$ is less than 2 and the poles of $g(\xi)$ are of multiplicity at least $j$.

Here, as usual, $g^{\#}(\xi)=\frac{\left|g^{\prime}(\xi)\right|}{1+|g(\xi)|^{2}}$ is the spherical derivative.

Lemma 2 ([17], Theorem 2). Let $f(z)$ be a transcendental meromorphic function in $\mathbb{C}$. If all zeros of $f(z)$ have multiplicity at least 3 , for any positive integer $k$, then $f^{(k)}(z)$ assumes every non-zero finite value infinitely often.

Lemma 3 ([17]). Let $f(z)=a_{n} z^{n}+a_{n-1} z^{n-1}+\cdots+a_{0}+q(z) / p(z)$, where $a_{0}, a_{1}, \cdots, a_{n}$ are constants with $a_{n} \neq 0$, and $q$ and $p$ are two co-prime polynomials, neither of which vanishes identically, with $\operatorname{deg} q<\operatorname{deg} p$; and let $k$ be a positive integer and $b$ a nonzero complex number. If $f^{(k)} \neq b$, and the zeros of $f$ all have multiplicity at least $k+1$, then

$$
f(z)=\frac{b(z-d)^{k+1}}{k !(z-c)},
$$

where $c$ and $d$ are distinct complex numbers.

Lemma 4 ([19], Lemma 4). Let $f$ be a nonconstant rational function and $k, m$ be positive integers. If $f$ has no zeros in $\mathbb{C}$ while all poles of $f$ have multiplicity at least $m$, then $f^{(k)}-1$ has at least $k+m$ distinct zeros in $\mathbb{C}$.

Lemma 5. Let $f$ be a non-constant meromorphic function, and $a \neq 0$ be a finite complex number. Let all poles of $f(z)$ have multiplicity at least 3 , then $f^{\prime}+a f^{2}$ has at least two distinct zeros. 
Proof. Set $f=\frac{1}{a \varphi}$, then all zeros of $\varphi$ have multiplicity at least 3 and $f^{\prime}+a f^{2}=-\frac{\varphi^{\prime}-1}{a \varphi^{2}}$.

Case 1. If $\frac{\varphi^{\prime}-1}{a \varphi^{2}}$ has only one zero $z_{0}$, then $z_{0}$ is a multiple pole of $\varphi$, or else a zero of $\varphi^{\prime}-1$. If $z_{0}$ is a multiple pole of $\varphi$, since $\frac{\varphi^{\prime}-1}{a \varphi^{2}}$ has only one zero, then $\varphi^{\prime}-1 \neq 0$. By Lemma 2 and Lemma 3 , this is a contradiction.

So $\varphi$ has no multiple pole and $\varphi^{\prime}-1$ has just one unique zero $z_{0}$. By the Lemma $2, \varphi$ is not any transcendental function.

Case 1.1. $\varphi$ is a non-constant polynomial.

Since $\varphi^{\prime}-1$ has only unique one zero $z_{0}$, set

$$
\varphi^{\prime}-1=A\left(z-z_{0}\right)^{l}
$$

where $A$ is non-zero constant, $l$ is a positive integer. Then

$$
\varphi^{\prime \prime}=A l\left(z-z_{0}\right)^{l-1}
$$

Note that $\varphi$ is a a non-constant polynomial and all zeros of $\varphi$ have multiplicity at least 3 , so $l \geqslant 3-1=2$. But $\varphi^{\prime \prime}$ has only one zero $z_{0}$, so $\varphi^{\prime}$ has only the same zero $z_{0}$ too. Hence $\varphi^{\prime}\left(z_{0}\right)=0$, which contradicts $\varphi^{\prime}\left(z_{0}\right)=1 \neq 0$. Therefore $\varphi$ is a rational function which is not a polynomial.

Case 1.2. If $\varphi$ is rational but not a polynomial and has at least one zero.

Under the conditions of Lemma 5 on the rational function $\varphi$,

$$
\varphi(z)=A \frac{\left(z+\alpha_{1}\right)^{m_{1}}\left(z+\alpha_{2}\right)^{m_{2}} \cdots\left(z+\alpha_{s}\right)^{m_{s}}}{\left(z+\beta_{1}\right)\left(z+\beta_{2}\right) \cdots\left(z+\beta_{t}\right)}
$$

where $A$ is a non-zero constant, $m_{i} \geqslant 3(i=1,2, \cdots, s), \alpha_{i}(i=1,2, \cdots, s)$, and $\beta_{j}(j=$ $1,2, \cdots, t)$ are distinct complex numbers.

For simplicity, we denote

$$
m_{1}+m_{2}+\cdots+m_{s}=M \geqslant 3 s .
$$

Let

$$
g(z)=z-\varphi(z)
$$

We use $\operatorname{deg}(g)$ to denote the degree of a polynomial. Next we use some results from complex dynamics, cf. [18, Chapter 3, 19 Lemma 5]. Since $\varphi^{\prime}-1$ has only unique one zero, so that $g$ has only unique one critical point. Since $m_{i} \geqslant 3$, we also see that every zero point $\alpha_{i}=-z_{i}$ of $-\varphi$ is a fixed point of $g$ of multiplicity $m_{i}$. Moreover, since near $z_{i}$

$$
g(z)=z+c_{i}\left(z-z_{i}\right)^{m_{i}}[1+o(1)]
$$


there are $m_{i}-1$ parabolic basins associated with the fixed point $z_{i}$.

Case 1.2.1. If $\infty$ is not a fixed point of $g$, each of these parabolic basins, with at most one exception, contains a critical point of $g$ which is not a pole of $g$, so the function $g(z)=z-\varphi(z)$ has $1 \geqslant M-s-1$ parabolic basins associated with the zero points of $\varphi$, we deduce that $s=1$ and $m_{1}=3$. We note that $\infty$ is not a fixed point of $g$, so

$$
\varphi(z)=\frac{\left(z+\alpha_{1}\right)^{3}}{\left(z+\beta_{1}\right)\left(z+\beta_{2}\right)}
$$

In order to simplify the calculation, set $Z=z+\beta_{1}$. From (2.3), we have

$$
\varphi(Z)=\frac{(Z+\alpha)^{3}}{Z(Z+\beta)}
$$

where $\alpha=\alpha_{1}-\beta_{1} \neq 0$ and $\beta=\beta_{2}-\beta_{1} \neq 0$.

We have

$$
\varphi^{\prime}=\frac{(Z+\alpha)^{2}\left(Z^{2}+2(\beta-\alpha) Z-\alpha \beta\right)}{Z^{2}(Z+\beta)^{2}}
$$

Since $\varphi^{\prime}(z)-1$ has exactly one zero $z_{0}$, from (2.3) we obtain

$$
\varphi^{\prime}(Z)=1+\frac{B\left(Z-Z_{0}\right)^{l}}{Z^{2}(Z+\beta)^{2}}=\frac{Z^{2}(Z+\beta)^{2}+B\left(Z-Z_{0}\right)^{l}}{Z^{2}(Z+\beta)^{2}}
$$

where $B$ is a non-zero constant and $Z_{0}=z_{0}-\beta_{1}$ and $l$ is a positive integer. From (2.4) and (2.5), we have $l \leq 3$.

From (2.4) we have

$$
\varphi^{\prime \prime}=2 \frac{(Z+\alpha)\left[\left(\beta^{2}+3 \alpha^{2}-3 \alpha \beta\right) Z^{2}+\left(3 \alpha^{2} \beta-\alpha \beta^{2}\right) Z+\alpha^{2} \beta^{2}\right]}{Z^{3}(Z+\beta)^{3}}
$$

From (2.5), we have

$$
\varphi^{\prime \prime}=B \frac{\left(Z-Z_{0}\right)^{l-1}\left[(l-4) Z^{2}+\left(l \beta+4 Z_{0}-2 \beta\right) Z+2 \beta Z_{0}\right]}{Z^{3}(Z+\beta)^{3}}
$$

If $l=1$, from (2.4) and (2.5), we have

$$
(Z+\alpha)^{2}\left(Z^{2}+2(\beta-\alpha) Z-\alpha \beta\right)=Z^{2}(Z+\beta)^{2}+B\left(Z-Z_{0}\right)
$$

By comparing coefficients of both sides, we have

$$
\left\{\begin{array}{c}
3 \alpha(\beta-\alpha)=\beta^{2} \\
-2 \alpha^{3}=B \\
-\alpha^{3} \beta=B Z_{0}
\end{array}\right.
$$


i.e.

$$
\left\{\begin{array}{c}
3 \alpha \beta-3 \alpha^{2}-\beta^{2}=0 \\
B=-2 \alpha^{3} \\
Z_{0}=\beta / 2
\end{array}\right.
$$

By (2.6), we have $\varphi^{\prime \prime}(-\alpha)=0$. From (2.7), we get

$$
(1-4)(-\alpha)^{2}+\left(\beta+4 \frac{\beta}{2}-2 \beta\right)(-\alpha)+2 \beta \frac{\beta}{2}=0
$$

i.e.

$$
\alpha \beta+3 \alpha^{2}-\beta^{2}=0
$$

Combining this with $3 \alpha \beta-3 \alpha^{2}-\beta^{2}=0$ yields

$$
\alpha=\beta=0
$$

which is a contradiction.

If $l=2$, from (2.4) and (2.5), we have

$$
(Z+\alpha)^{2}\left(Z^{2}+2(\beta-\alpha) Z-\alpha \beta\right)=Z^{2}(Z+\beta)^{2}+B\left(Z-Z_{0}\right)^{2}
$$

By comparing coefficients of both sides, we have

$$
\left\{\begin{array}{c}
3 \alpha(\beta-\alpha)=\beta^{2}+B \\
-2 \alpha^{3}=-2 B Z_{0} \\
-\alpha^{3} \beta=B Z_{0}^{2}
\end{array}\right.
$$

i.e.

$$
\left\{\begin{array}{c}
3 \alpha \beta^{2}-3 \alpha^{2} \beta-\beta^{3}+\alpha^{3}=0 \\
B=-\frac{\alpha^{3}}{\beta} \\
Z_{0}=-\beta
\end{array}\right.
$$

By (2.6), we have $\varphi^{\prime \prime}(-\alpha)=0$. From (2.7), we get

$$
\begin{aligned}
\varphi^{\prime \prime}(-\alpha) & =-\frac{\alpha^{3}}{\beta} \frac{(\beta-\alpha)\left[(2-4)(-\alpha)^{2}+(2 \beta-4 \beta-2 \beta)(-\alpha)-2 \beta^{2}\right]}{(-\alpha)^{3}(\beta-\alpha)^{3}} \\
& =-\frac{2}{\beta} \neq 0
\end{aligned}
$$

which is a contradiction. If $l=3$, then $\operatorname{deg}\left((Z+\alpha)\left[\left(\beta^{2}+3 \alpha^{2}-3 \alpha \beta\right) Z^{2}+\left(3 \alpha^{2} \beta-\alpha \beta^{2}\right) Z+\right.\right.$ $\left.\left.\alpha^{2} \beta^{2}\right]\right)<\operatorname{deg}\left(\left(Z-Z_{0}\right)^{l-1}\left[(l-4) Z^{2}+\left(l \beta+4 Z_{0}-2 \beta\right) Z+2 \beta Z_{0}\right]\right)$, which is a contradiction.

Case 1.2.2. If $\infty$ is a fixed point of $g$, each parabolic basin contains a critical point of $g$ which is not a pole of $g$. Thus each parabolic basins contains a zero point of $g^{\prime}$, and hence $2 \leq M-s \leq 1$, which is a contradiction. 
Case 1.3. If $\phi$ is rational but not a polynomial and has no zero. By Lemma 4 , we have $\varphi^{\prime}-1$ has at least two distinct zeros, which contradicts the fact that $\varphi^{\prime}-1$ has just one unique zero $z_{0}$.

Case 2. If $\frac{\varphi^{\prime}-1}{a \varphi^{2}} \neq 0$.

Case 2.1. Since all zeros of $\varphi(z)$ have the multiple at least 3 and $\varphi$ is a non-constant function, it easily obtained that $\varphi$ is not a polynomial.

Case 2.2. If $\varphi$ is rational but not a polynomial. If there exist a point $z_{0}$ such that $\varphi^{\prime}\left(z_{0}\right)=1$, we note that $\frac{\varphi^{\prime}-1}{a \varphi^{2}} \neq 0$, so $\varphi\left(z_{0}\right)=0$. Since all zeros of $\varphi(z)$ have the multiple at least 3 , we have $\varphi^{\prime}\left(z_{0}\right)=0$, we get a contradiction.

If $\varphi^{\prime} \neq 1$, by Lemma 3 , we have

$$
\varphi=\frac{(z-d)^{2}}{(z-c)}
$$

where $c$ and $d$ are distinct complex numbers. This contradicts the fact that all zeros of $\varphi(z)$ have the multiple at least 3.

The proof is complete.

\section{Proofs of Theorems}

Proof of Theorem 1. Suppose that $\mathscr{F}$ is not normal in $\mathscr{D}$. Then there exists at least one point $z_{0}$ such that $\mathscr{F}$ is not normal at the point $z_{0} \in \mathscr{D}$. Without loss of generality we assume that $z_{0}=0$. By Zalcman's lemma, there exist:

(a) points $z_{n}, z_{n} \rightarrow z_{0}$;

(b) functions $f_{n} \in \mathscr{F}$; and

(c) positive numbers $\rho_{n} \rightarrow 0^{+}$

such that

$$
g_{j}(\xi)=\rho_{j} f_{j}\left(z_{j}+\rho_{j} \xi\right) \rightarrow g(\xi)
$$

spherically uniformly on compact subsets of $\mathbb{C}$, where $g(\xi)$ is a non-constant meromorphic function in $\mathbb{C}$ and the poles of $g(\xi)$ are of multiplicity at least 3 .

From (3.1), we get

$$
g_{j}^{\prime}(\xi)=\rho_{j}^{2} f_{j}^{\prime}\left(z_{j}+\rho_{j} \xi\right) \rightarrow g^{\prime}(\xi)
$$

also locally uniformly with respect to the spherical metric.

Thus

$$
g_{j}^{\prime}(\xi)+a g_{n_{j}}(\xi)-\rho_{j}^{2} b=\rho_{j}^{2}\left(f_{j}^{\prime}\left(z_{j}+\rho_{j} \xi\right)+a f_{j}^{2}\left(z_{j}+\rho_{j} \xi\right)-b\right) \rightarrow g^{\prime}(\xi)+a g^{2}(\xi)
$$


also locally uniformly with respect to the spherical metric.

We claim that $g^{\prime}(\xi)+a g^{2}$ has at most 1 zero ignoring multiplicity.

If $g^{\prime}(\xi)+a g^{2} \equiv 0$, then $g(\xi) \equiv \frac{1}{a z+c}$, this contradicts the fact that the poles of $g(\xi)$ are of multiplicity at least 3 . So $g^{\prime}(\xi)+a g^{2} \not \equiv 0$.

Suppose that $g^{\prime}(\xi)+a g^{2}$ has two distinct zeros $\xi_{0}$ and $\xi_{0}^{*}$ and choose $\delta(>0)$ small enough such that $D\left(\xi_{0}, \delta\right) \cap D\left(\xi_{0}^{*}, \delta\right)=\varnothing$ where $D\left(\xi_{0}, \delta\right)=\left\{\xi|| \xi-\xi_{0} \mid<\delta\right\}$ and $D\left(\xi_{0}^{*}, \delta\right)=\left\{\xi|| \xi-\xi_{0}^{*} \mid<\delta\right\}$.

From (3.3), by Hurwitz's theorem, there exist points $\xi_{j} \in D\left(\xi_{0}, \delta\right), \xi_{j}^{*} \in D\left(\xi_{0}^{*}, \delta\right)$ such that for sufficiently large $j$

$$
\begin{aligned}
& f_{j}^{\prime}\left(z_{j}+\rho_{j} \xi_{j}\right)+a f_{j}^{2}\left(z_{j}+\rho_{j} \xi_{j}\right)-b=0 . \\
& f_{j}^{\prime}\left(z_{j}+\rho_{j} \xi_{j}^{*}\right)+a f_{j}^{2}\left(z_{j}+\rho_{j} \xi_{j}^{*}\right)-b=0 .
\end{aligned}
$$

Since $z_{j} \rightarrow 0$ and $\rho_{j} \rightarrow 0^{+}$, we have $z_{j}+\rho_{j} \xi_{j} \in D\left(\xi_{0}, \delta\right)$ and $z_{j}+\rho_{j} \xi_{j}^{*} \in D\left(\xi_{0}^{*}, \delta\right)$ for sufficiently large $j$, so $f_{j}^{\prime}(z)+a f_{j}^{2}-b$ has two distinct zeros, which contradicts the fact that $f_{j}^{\prime}(z)+a f_{j}^{2}-b$ has at most 1 zero.

However, by Lemma 5, there does not exist non-constant meromorphic functions satisfying above properties such that our claim holds. This contradiction shows that $\mathscr{F}$ is normal in $\mathscr{D}$ and hence Theorem 1 is proved.

Proof of Theorem 2. Suppose that $\mathscr{F}$ is not normal in $\mathscr{D}$. Then there exists at least one point $z_{0}$ such that $\mathscr{F}$ is not normal at the point $z_{0} \in \mathscr{D}$. Without loss of generality we assume that $z_{0}=0$. By Zalcman's lemma, there exist:

(a) points $z_{n}, z_{n} \rightarrow z_{0}$;

(b) functions $f_{n} \in \mathscr{F}$; and

(c) positive numbers $\rho_{n} \rightarrow 0^{+}$

such that

$$
g_{j}(\xi)=\rho_{j} f_{j}\left(z_{j}+\rho_{j} \xi\right) \rightarrow g(\xi)
$$

spherically uniformly on compact subsets of $\mathbb{C}$, where $g(\xi)$ is a non-constant meromorphic function in $\mathbb{C}$ and the poles of $g(\xi)$ are of multiplicity at least 3 .

Proceeding as in the proof of Theorem 1, we also have (3.3).

If $g^{\prime}(\xi)+a g^{2} \equiv 0$, then $g(\xi) \equiv \frac{1}{a z+c}$, this contradicts the fact that the poles of $g(\xi)$ are of multiplicity at least 3 . So $g^{\prime}(\xi)+a g^{2} \not \equiv 0$.

Since $g$ is a non-constant meromorphic function, by Lemma 5 , we deduce that $g^{\prime}(\xi)+a g^{2}$ has at least two distinct zeros.

We claim that $g^{\prime}(\xi)+a g^{2}(\xi)$ has just a unique zero. 
Suppose that there exist two distinct zeros $\xi_{0}$ and $\xi_{0}^{*}$ and choose $\delta(>0)$ small enough such that $D\left(\xi_{0}, \delta\right) \cap D\left(\xi_{0}^{*}, \delta\right)=\varnothing$ where $D\left(\xi_{0}, \delta\right)=\left\{\xi|| \xi-\xi_{0} \mid<\delta\right\}$ and $D\left(\xi_{0}^{*}, \delta\right)=\left\{\xi|| \xi-\xi_{0}^{*} \mid<\delta\right\}$.

From (3.4), by Hurwitz's theorem, there exist points $\xi_{j} \in D\left(\xi_{0}, \delta\right), \xi_{j}^{*} \in D\left(\xi_{0}^{*}, \delta\right)$ such that for sufficiently large $j$

$$
\begin{aligned}
& f_{j}^{\prime}\left(z_{j}+\rho_{j} \xi_{j}\right)+a f_{j}^{2}\left(z_{j}+\rho_{j} \xi_{j}\right)-b=0 . \\
& f_{j}^{\prime}\left(z_{j}+\rho_{j} \xi_{j}^{*}\right)+a f_{j}^{2}\left(z_{j}+\rho_{j} \xi_{j}^{*}\right)-b=0 .
\end{aligned}
$$

By the assumption that $f^{\prime}+a f^{2}$ and $g^{\prime}+a g^{2}$ share $b$ in $\mathscr{D}$ for each pair $f$ and $g$ in $\mathscr{F}$, we know that for any integer $m$

$$
\begin{aligned}
& f_{m}^{\prime}\left(z_{j}+\rho_{j} \xi_{j}\right)+a f_{m}^{2}\left(z_{j}+\rho_{j} \xi_{j}\right)-b=0 . \\
& f_{m}^{\prime}\left(z_{j}+\rho_{j} \xi_{j}^{*}\right)+a f_{m}^{2}\left(z_{j}+\rho_{j} \xi_{j}^{*}\right)-b=0 .
\end{aligned}
$$

We fix $m$ and note that $z_{j}+\rho_{j} \xi_{j} \rightarrow 0, z_{j}+\rho_{j} \xi_{j}^{*} \rightarrow 0$ if $j \rightarrow \infty$. From this we deduce

$$
f_{m}^{\prime}(0)+a f_{m}^{2}(0)-b=0 .
$$

Since the zeros of $f_{m}^{\prime}(z)+a f_{m}^{2}(z)-b$ have no accumulation point, for sufficiently large $j$, we have

$$
z_{j}+\rho_{j} \xi_{j}=0, z_{j}+\rho_{j} \xi_{j}^{*}=0
$$

Hence

$$
\xi_{j}=-\frac{z_{j}}{\rho_{j}}, \xi_{j}^{*}=-\frac{z_{j}}{\rho_{j}} .
$$

This contradicts the fact that $\xi_{j} \in D\left(\xi_{0}, \delta\right), \xi_{j}^{*} \in D\left(\xi_{0}^{*}, \delta\right)$ and $D\left(\xi_{0}, \delta\right) \cap D\left(\xi_{0}^{*}, \delta\right)=\varnothing$. So $g^{\prime}(\xi)+a g^{2}(\xi)$ has just a unique zero. This contradicts the fact that $g^{\prime}(\xi)+a g^{2}(\xi)$ has at least two distinct zeros.

This proves the theorem.

\section{Acknowledgement}

This work was supported by Foundation of Shaanxi Railway Institute (Grant No. 201312). The authors thank the reviewer(s) for reading the manuscript very carefully and making a number of valuable and kind comments which improved the presentation. 


\section{References}

[1] J. Schiff, Normal Families, Springer-Verlag, Berlin, 1993.

[2] C. C. Yang and H. X. Yi, Uniqueness Theory of Meromorphic Functions, Science Press, Beijing, 1995 (In Chinese); Kluwer Academic Publishers, The Netherlands, 2003.

[3] W. K. Hayman, Picard values of meromorphic functions and their derivatives, Ann. of Math., 70(1959), 9-42.

[4] W. K. Hayman, Research Problems in Function Theory, Athlone Press, London, 1967.

[5] S. Li, On normality criterion of a class of the functions, J. Fujian Normal Univ., 2 (1984), 156-158 (in Chinese).

[6] X. Li, Proof of Hayman's conjecture on normal families, Sci. China Ser. A, 28 (1985), 596-603.

[7] J. Langley, On normal families and a result of Drasin, Proc. Roy. Soc. Edinburgh Sect. A, 98 (1984), $385-393$.

[8] X. Pang, On normal criterion of meromorphic functions, Sci. China Ser. A, 33 (1990), 521-527.

[9] H. Chen and M. Fang, On the value distribution of $f^{n} f^{\prime}$, Sci. China Ser. A, 38 (1995), 789-798.

[10] L. Zalcman, On some questions of Hayman, unpublished manuscript, 1994.

[11] D. Drasin, Normal families and Nevanlinna theory, Acta Math., 122 (1969), 231-263.

[12] Y. Ye, A new criterion and its application, Chinese Ann. Math. Ser. A, 12 (suppl.) (1991), 44-49 (in Chinese).

[13] M. L. Fang and W. J. Yuan, On the normality for families of meromorphic functions, Indian J. Math., 43(2001), 341-350.

[14] Q. Zhang, Normal families of meromorphic functions concerning shared values, J. Math. Anal. Appl., 338(2008), 545-551.

[15] L. Zalcman, Normal families: New perspectives, Bull. Amer. Math. Soc., 35 (1998), 215-230.

[16] W. J. Yuan and M. L. Fang, Further results of the normality for meromorphic function families, J. Guangzhou University (Natural Science Edition), 1(2002), 1-9.

[17] Y. F. Wang and M. L. Fang, Picard values and normal families of meromorphic functions with multiple zeros, Acta Math. N. S., 14(1998), 17-26. .

[18] N. Steinmetz, Rational iteration, De Gruyter Studies in Mathematics 16, Walter de Gruyter, Berlin, New Youk, 1993.

[19] J. M. Chang, Normality of meromorphic functions whose derivatives have 1-points, Arch. Math., 94(2010), 555-564.

Department of Basic Courses, Shaanxi Railway Institute, Weinan 714000, Shaanxi Province, PR China.

E-mail: liyuntong2005@sohu.com 